\title{
Inteligencia y creatividad
}

\author{
Ferrando, M. ${ }^{1}$, Prieto, M.D. ${ }^{1}$, Ferrándiz, C. ${ }^{1}$ \\ y Sánchez, C. $^{2}$
}

\footnotetext{
${ }^{1}$ Departamento de Psicología Evolutiva y de la Educación. Facultad de Psicología. Universidad de Murcia, España

${ }^{2}$ Departamento de Métodos de Investigación y Diagnóstico en Educación. Facultad de Educación. Universidad de Murcia, España
}

\section{España}

mferran@um.es 


\section{Resumen}

Introducción Han sido numerosos los autores que han investigado la relación existente entre la creatividad y la inteligencia, siendo diversos los resultados encontrados. Así Guilford (1950) incluye la creatividad dentro del constructo inteligencia, Sternberg (1988) alude a que la creatividad englobaría al constructo inteligencia; Gardner (1995) apunta a la estrecha relación entre la creatividad y el dominio en el cual se manifiesta una determinada inteligencia; y para Getzels y Jackson (1962) y Torrance (1962) la inteligencia y la creatividad serían independientes. El objetivo del trabajo es doble: por una parte, establecer la relación entre creatividad e inteligencia general; por otra, estudiar la relación existente entre la creatividad y las Inteligencias Múltiples (IM).

Método: Primero, presentamos un breve recorrido histórico sobre el concepto de la creatividad. Segundo, estudiamos la perspectiva de las Inteligencias Múltiples y Creatividad Múltiple de Gardner. Tercero, analizamos los datos procedentes del estudio empírico, realizado con 294 alumnos ( $3^{\circ}$ de Educación Infantil y $1^{\circ}$ y $2^{\circ}$ de Educación Primaria).

Resultados: Los datos indican que no existe relación entre la inteligencia y la creatividad; se constata la teoría del umbral propuesta por Torrance para las IM; en el sentido de que cierto nivel intelectual, es necesario aunque no suficiente para la creatividad. La relación entre la creatividad y las Inteligencias Múltiples (IM) es más elevada que entre la psicométrica y la creatividad; siendo la inteligencia viso-espacial la que mayor relación guarda con la creatividad, seguida de la naturalista, corporal y lingüística.

Discusión: los resultados indican bajas relaciones entre creatividad e inteligencia. No obstante, la relación entre ellos cambia según se conceptualice el constructo de inteligencia. En general, podríamos decir que existe una mayor relación entre la creatividad y las inteligencias múltiples. Asimismo, no se constata la teoría del umbral cuando se relaciona el CI con la creatividad: los alumnos con un mayor Cociente Intelectual no son los más creativos. Sin embargo, desde la perspectiva multidimensional la teoría del umbral se corrobora para la inteligencia viso-espacial, corporal, naturalista y lingüística;

Palabras Clave: inteligencia, creatividad, inteligencias múltiples, evaluación, evaluación inteligencia psicométrica. 


\section{Introducción}

La relación entre la creatividad y la inteligencia es un tópico que, a pesar de haber sido estudiado desde sus inicios, sigue sin estar claro. Tradicionalmente se había asumido que las personas creativas eran también personas inteligentes. Sin embargo, hay autores que no tienen tan claro que se trate de la misma realidad; de hecho dicen que si son la misma cosa, podemos asumir que una persona creativa es forzosamente inteligente y viceversa, si una persona es inteligente, será creativa. O podemos tomar las palabras de Wallach y Kogan (1965), quienes dicen que, al igual que se puede demostrar la existencia de diferentes grados de aptitud cognitiva o inteligencia, también se pueden establecer niveles de creatividad, pero como algo independiente de la inteligencia, y que con justicia se puede llamar «creatividad».

A pesar de que algunos hayan defendido la postura de que para producir obras creadoras significativas es necesario poseer un alto nivel de inteligencia, lo cierto es que la alta inteligencia no garantiza la actividad creadora y, por supuesto, la baja inteligencia es seguro que no ayuda; por ello, muchos psicólogos se inclinaron por la tesis de que inteligencia y creatividad son dos capacidades independientes. Además, esta hipótesis se veía reforzada por el hecho de que se comprobó la existencia de bajas correlaciones entre inteligencia y rendimiento académico. Este hecho sirvió para deducir que además del rendimiento escolar, influían en la creatividad otros factores distintos a la inteligencia. A partir de aquí, comenzaron a diferenciarse cinco hipótesis de trabajo:

El primer planteamiento es el hecho por Guilford (1950), quien argumenta que, si bien no se encuentra esta relación (inteligencia y creatividad), o se da en un grado bajo, esto es debido a que las pruebas de inteligencia no miden todas las aptitudes de la inteligencia, entre las que se encuentran las directamente implicadas en la creatividad. Con esta explicación Guilford no estaba asumiendo que la creatividad fuese cosa distinta de la inteligencia, sino más bien que una parte de ésta (el pensamiento divergente) no estaba siendo medida en los tests estandarizados de inteligencia. Por tanto, estaba asumiendo que la inteligencia es un constructo amplio en el cual se enmarca la creatividad

Un segundo planteamiento es el realizado por quienes se han decantado por asumir otro tipo de relación entre ambos constructos, sosteniendo que se trata de realidades independientes "dos partes de un mismo conjunto que encajan la una en la otra" (Wallach y Kogan, 
1965) Esta hipótesis supone, por tanto, que la creatividad y la inteligencia son rasgos independientes, son dos variables independientes y distintas, no encontrándose relación entre las mismas, o si ésta se da es sólo en edades determinadas o entre CI concretos.

Las investigaciones que mejor han puesto de manifiesto esta afirmación son, por un lado, la de Gelzels y Jackson (1962), quienes presentaron el primer estudio que trataba de investigar la relación entre creatividad e inteligencia. Su investigación, aunque ha servido como modelo a partir de la cual se han llevado a cabo otras muchas, no quedó en absoluto exenta de críticas. Su investigación se hizo en un colegio privado, cuyos alumnos presentaban un Cociente Intelectual muy elevado (CI: 132) cosa que posteriormente sería criticada por diferentes autores. La investigación trató de discernir entre los niños muy inteligentes (seleccionaron un grupo de 28 niños) y los niños muy creativos (seleccionaron un grupo de 26 alumnos). Una de las conclusiones a la que llegaron los autores fue que la correlación entre creatividad e inteligencia era muy baja, lo que equivale a decir que se trataba de dos entidades diferentes. Las críticas de la metodología que se había utilizado en la investigación posibilitaron réplicas que ayudaron a subsanar estos fallos (Alonso, 2000).

Fue Torrance (1962) quien, partiendo también del criterio de que la creatividad y la inteligencia eran constructos distintos, reproduce el mismo esquema de investigación que Getzels y Jackson en ocho escuelas, constatando que el grupo de alta creatividad tenía el mismo rendimiento escolar que el grupo de alto CI, excepto en una escuela parroquial y en una escuela primaria de una pequeña ciudad. Él explicó estos resultados mediante la "teoría del umbral", que explica las relaciones entre inteligencia y creatividad postulando que, cuando el CI está por debajo de un cierto límite, la creatividad también se encuentra limitada, mientras que cuando el CI se sitúa por encima de este límite (CI: 115-120) la creatividad llega a ser una dimensión casi independiente del CI. Dicho de otra forma: un cierto nivel intelectual es condición necesaria, pero no suficiente, para el desarrollo de la creatividad.

Otra de las investigaciones más prestigiosas fue la llevada a cabo por Wallach y Kogan (1965), quienes perfeccionaron y depuraron los fallos cometidos en las investigaciones anteriores de sus colegas. Parten de la idea de que la creatividad debe ser considerada como una manera de pensar diferente a la inteligencia. Adoptan la definición de proceso creador realizada por Mednick (1963), sosteniendo que una buena medida de creatividad es el número y el carácter único de asociaciones relevantes que se pueden producir en una situación abierta, 
y tratan de ver si así se puede mantener la distinción entre inteligencia y creatividad, y en qué medida los distintos grupos de sujetos que se forman combinando niveles de inteligencia y creatividad diferirían respecto a su comportamiento escolar, sus juegos y su personalidad. En esta investigación se establecían cuatro grupos diferenciados de sujetos, según las combinaciones de creatividad-inteligencia que podían encontrarse. A saber: a) sujetos muy inteligentes y muy creativos, b) sujetos muy inteligentes pero poco creativos, c) sujetos poco inteligentes pero muy creativos, d) sujetos poco inteligentes y poco creativos. Los sujetos pertenecientes a cada uno de estos grupos presentaban diferencias en cuanto a los otros. Los datos procedentes de los estudios confirmaban que la diferencia entre ambas variables se manifestaban de forma diferente y que se podían encontrar combinadas de todas las formas posibles.

Un tercer planteamiento es el hecho por aquellos autores que han destacado que, si bien ambos constructos parecen independientes, no se puede negar que están fuertemente relacionados entre sí, superponiéndose en algunos casos. Estudiando las conductas entre los inteligentes y los creativos, observaron que determinados efectos podían atribuirse a una (inteligencia) o a otra (creatividad). Esto aparecía fundamentalmente en el análisis de la solución de problemas. Si la creatividad es una manera de resolver problemas, no cabe duda de que la inteligencia es necesaria para resolverlos; cuando la solución precisa niveles muy altos de inteligencia, será necesario un comportamiento creativo. Sobre esta idea ha habido aportaciones muy diferentes:

El instituto para la evaluación e investigación de la personalidad (IPAR, Institute Personality Assessment Research) se dedicó, entre otras cosas, al estudio de la personalidad y a la investigación sobre la relación entre inteligencia y creatividad. En dicho instituto se hicieron los estudios referidos a la relación existente entre creatividad, inteligencia y personalidad (Barron y Harrington, 1981; McKinnon, 1962, 1970; Barron, 1988), quienes desarrollaron un trabajo en el cual intentaban poner de relieve cuál era el CI de los creativos; para ello, trabajaron con personas muy creativas vivas (arquitectos, deportistas, científicos, matemáticos, etc.), que fueron seleccionadas como tales por expertos. Se les convocó durante unos fines de semana a convivencias en las que se les administraron tests de inteligencia, creatividad y personalidad, se les sometió a pruebas situacionales y de grupo, todo bajo la observación de unos siete observadores por cada diez seleccionados. Los datos se analizaron comparando los datos de los grupos estudiados con los datos de otros profesionales equiparados en todo menos en la creatividad. Sus hallazgos respondían a la pregunta de cuál era el CI de los creativos. Los 
datos reflejaron que la correlación entre CI y creatividad no era idéntica en todos los creativos, pues aparecían correlaciones débiles y moderadas. Sin embargo, no tendían a aparecer correlaciones muy altas, aunque esto dependía mucho del campo de producción del creativo (no era la misma correlación media en arte que en ciencias), debido fundamentalmente al diferente papel que en cada campo juega la inteligencia. Aun así, los altamente creativos, tendían a tener un CI por encima de la media, aunque un CI por encima de 120 puntos no parecía tener influencia en el aumento de la creatividad.

La investigación de Renzulli (1977) también se encuadra dentro de esta perspectiva. Dice el autor que la creatividad y la inteligencia son realidades distintas, que en determinadas circunstancias se superponen, pudiéndose hallar juntas. Él propone su modelo de los tres anillos. Estos tres anillos son la "creatividad" "la inteligencia" y "la persistencia en la tarea". Éstos pueden hallarse por separado, o también juntos, de tal manera que el altamente creativo suele producir muchas ideas, mientras que el altamente persistente coincide con el niño que convencionalmente se aprende las lecciones de memoria.

Mednick (1963) es otro autor que defiende la estrecha relación entre creatividad e inteligencia. Según él, la creatividad consiste en asociaciones que, cuanto más lejanas, más creativas. Ahora bien, la causa de la habilidad para hacer estas combinaciones y llegar a soluciones creativas depende, necesariamente, de la existencia de una especie de almacén de conocimientos que permite hacer combinaciones y lograr una cierta velocidad para llegar a una solución creativa, que está influenciada por la organización de las asociaciones de las personas. La teoría de Mednick sugiere que creatividad e inteligencia están muy relacionadas. Sus estudios sobre correlación entre medidas psicométricas y medidas de la creatividad evaluadas por su test (RAT, Remote Asociation Test) han establecido una correlación de 0.55 entre el WISC (Weschler Intelligence Scale) y el RAT, siendo la correlación entre el SAT (School Aptitude Test) y el RAT de 0.43 ; sin embargo, las correlaciones entre el RAT y otras medidas de creatividad son más variables.

Un cuarto planteamiento es el que sostiene que la creatividad es un constructo más amplio que abarca a la inteligencia. Esta es la hipótesis defendida por Sternberg (1988). De hecho, es uno de sus estudios sobre la concepción que tenían las personas sobre inteligencia y creatividad pasó un cuestionario a diferentes personas y los resultados demostraron que la percepción de las personas sobre estos dos constructos es la misma, pues la mayoría atribuían 
las mismas características a los creativos y a los inteligentes; es decir, en la calle se asume la inteligencia y la creatividad como una única realidad.

Entre las investigaciones que apoyan esta hipótesis que sostiene que la inteligencia y la creatividad son la misma cosa, tenemos la realizada por Weisberg y Alba (1981) y Weisberg (1988), quienes han argumentado que los mecanismos para la creatividad no son diferentes de los mecanismos usados en la solución de problemas. De acuerdo con estos investigadores, un trabajo es juzgado como creativo cuando los procesos ordinarios de pensamiento producen resultados extraordinarios. Por tanto, si queremos entender la creatividad, no necesitamos ir más allá del estudio de los procesos ordinarios que se utilizan en la solución de problemas (Sternberg y O’Hara, 1999). Para demostrar esta hipótesis, Weisberg (1988) utilizó el ya conocido problema de los nueve puntos, que consiste en pedirle al individuo que trate de unir todos los puntos sin levantar el lápiz del papel. Normalmente la solución a este problema se da por insigth cuando el sujeto dsecubre que puede salir fuera de la estructura cerrada de los puntos; es decir, las líneas pueden rebasar los puntos. Ya anteriormente Weisberg y Alba (1981) demostraron que, aunque las personas hubieran tenido este insight, seguían teniendo dificultades para resolver el problema.

En definitiva, los defensores de esta postura piensan que tanto la inteligencia como la creatividad forman parte de una realidad integrada; es decir, argumentan que son en realidad dos facetas de una misma función singular y única que se origina en la capacidad mental: creatividad e inteligencia son expresiones de la capacidad mental, pero cada una tiene una finalidad distinta y exige unos recursos diferentes, lo que significa que ambas atienden a la información, la recuperan y la organizan de manera diferente. Pero ambas contribuyen a dar una respuesta integrada que se adapta a una tarea especifica o al ambiente inmediato (Genovard, Prieto, Bermejo y Ferrándiz, en prensa).

Ahora bien, desde 1983 una nueva concepción de la inteligencia está siendo discutida en el campo de la psicología. Es la perspectiva de las Inteligencias Múltiples formulada por Gardner, de quien recogemos sus principios sobre la creatividad. Este autor ha dejado de ver la inteligencia como algo unitario y compacto, diversificando las inteligencias. Según sus planteamientos teóricos, creatividad e inteligencia son lo mismo; de hecho, sus definiciones de persona creativa y persona inteligente son idénticas. Él plantea que la creatividad se apoya en lo que llama "pacto faustiano", por el que se renuncia a lo fácil y agradable para conseguir 
objetivos determinados, es decir que la creatividad sería un impulso de persistencia en el esfuerzo para seguir sus metas. A continuación comentamos los principios de la teoría por ser el modelo que ponemos a prueba en el estudio empírico.

\section{Inteligencias Múltiples, creatividad y contexto}

Tradicionalmente hemos pensado e incluso aceptado que la inteligencia es una capacidad unitaria que abarca varias capacidades. Sin embargo, en oposición a este enfoque más bien reduccionista, Gardner (1983) propone su teoría de las Inteligencias Múltiples (IM). Se trata de un planteamiento sugerente, e incluso provocador, que permite cuestionar el constructo de la inteligencia más allá de lo cognitivo.

Para este autor la inteligencia es la capacidad de resolver problemas o de crear productos que sean valiosos en uno o más ambientes culturales. El punto crítico de su teoría consiste en reconocer la existencia de ocho inteligencias diferentes e independientes, que pueden interactuar y potenciarse recíprocamente. La existencia de una de ellas, sin embargo, no predice la existencia de alguna de las otras. Las inteligencias propuestas son: lingüística, lógicomatemática, espacial, musical, corporal-kinestésica, interpersonal, intrapersonal y naturalista.

De forma breve podemos definir las inteligencias de este modo:

- Inteligencia Lingüística: es la capacidad implícita en la lectura y escritura, así como en el escuchar y hablar. Comprende la sensibilidad para los sonidos y las palabras con sus matices de significado, su ritmo y sus pausas. Está relacionada con el potencial para estimular y persuadir por medio de la palabra.

- Inteligencia Lógico-Matemática: es la capacidad relacionada con el razonamiento abstracto, la computación numérica, la derivación de evidencias y la resolución de problemas lógicos.

- Inteligencia Espacial: es la capacidad utilizada para enfrentar problemas de desplazamiento y orientación en el espacio, reconocer situaciones, escenarios o rostros. Permite crear modelos del entorno viso-espacial y efectuar transformaciones a partir de él, aun en ausencia de los estímulos concretos.

- Inteligencia Musical: es la capacidad para producir y apreciar el tono, ritmo y timbre de la música. Se expresa en el canto, la ejecución de un instrumento, la composición, la dirección orquestal o la apreciación musical. 
- Inteligencia Corporal: es la capacidad para utilizar el propio cuerpo ya sea total o parcialmente, en la solución de problemas o en la interpretación. Implica controlar los movimientos corporales, manipular objetos y lograr efectos en el ambiente.

- Inteligencia Interpersonal: es la capacidad para entender a los demás y actuar en situaciones sociales, para percibir y discriminar emociones, motivaciones o intenciones. Está estrechamente asociada a los fenómenos interpersonales como la organización y el liderazgo.

- Inteligencia Intrapersonal: es la capacidad para comprenderse a sí mismo, reconocer los estados personales, las propias emociones, tener claridad sobre las razones que llevan a reaccionar de un modo u otro, y comportarse de una manera que resulte adecuada a las necesidades, metas y habilidades personales. Permite el acceso al mundo interior para luego poder aprovechar y a la vez orientar la experiencia.

- Inteligencia Naturalista: es la capacidad para percibir las relaciones que existen entre varias especies o grupos de objetos y personas, así como reconocer y establecer si existen distinciones y semejanzas entre ellos. Implica habilidades para observar, identificar y clasificar a los miembros de un grupo o especie, e incluso para descubrir nuevas especies.

Así pues, hablar de inteligencias múltiples, y concebirlas como sistemas independientes, replantea la discusión sobre los factores de la inteligencia, habitualmente asociados a una dimensión cognitiva. Ciertamente, también cambia cualquier perspectiva sobre estimulación y desarrollo de esta capacidad. En síntesis, Gardner nos conduce a redimensionar la importancia de los componentes racionales, invitándonos a reconocer y valorar otras expresiones de la persona que no guardan relación directa con logros cognitivos.

\section{Inteligencias Múltiples y creatividad Múltiple}

Gardner postula que los seres humanos son capaces de conocer y de aprender de ocho maneras diferentes: a través del lenguaje, del análisis lógico-matemático, de la representación espacial, del pensamiento musical, del uso del cuerpo, de la observación y conocimiento del mundo que nos rodea, de una comprensión de los demás y de nosotros mismos. Todos los individuos pueden presentar estas inteligencias, pero claramente en distinta intensidad, y con diferencias en las formas en que se recurre a ellas y se las combina para llevar a cabo determinadas tareas. En la vida cotidiana las inteligencias operan en armonía, dentro de un cierto perfil de inteligencias, de modo que no es fácil reconocer su autonomía e independencia, pero cuando se observa con atención, la naturaleza peculiar de cada una se hace evidente. 
El reconocimiento de esta diversidad hace más compleja la comprensión de la experiencia y el aprendizaje humano. Estas diferencias desafían un sistema educativo que supone que todos pueden aprender las mismas cosas del mismo modo, y que basta con una medida uniforme y universal para poner a prueba a un estudiante. En los debates sobre inteligencia y educación es preciso tomar en cuenta estas distintas formas del pensamiento, así como los contextos en los que es posible expresarlas o desarrollarlas.

Gardner postula que inteligencia y creatividad no deben comprenderse como fenómenos separados. Rompe así una dicotomía de larga presencia entre los estudiosos de la creatividad. El primer paso consiste en reemplazar la pregunta convencional: ¿Qué es la creatividad?, por otra que definitivamente cambia la dirección de la búsqueda: ¿Dónde está la creatividad? La primera todavía permite una respuesta más abstracta, sin contexto, en tanto que la segunda impone la obligación de una mirada más amplia. En el curso de este movimiento surge la siguiente propuesta: Individuo creativo es la persona que resuelve problemas con regularidad, elabora productos o define cuestiones nuevas en un campo de un modo que al principio es considerado original, pero que al final llega a ser aceptado en un contexto cultural concreto (1993). Gardner caracteriza la creatividad y la persona creativa del siguiente modo:

La creatividad implica novedad inicial y aceptación final.

La creatividad se caracteriza por la elaboración de nuevos productos o el planteamiento de nuevos problemas.

Las actividades creativas sólo son conocidas como tales cuando han sido aceptadas en una cultura concreta.

Una persona suele ser creativa en un campo y no en todos.

Una persona es creativa cuando manifiesta su creatividad en forma consistente.

Si la inteligencia es plural, lo es también la creatividad. Gardner quiere demostrar el carácter distintivo de las actividades habituales de una persona creativa. Así como no hay un tipo único de inteligencia, tampoco puede haber un tipo único de creatividad. Este es el fallo de los tests de creatividad: en su ingenua suposición de que el desempeño acertado frente a tareas divergentes y muy triviales garantiza una predicción respecto a comportamientos futuros en cualquier campo. 
En su libro Mentes Creativas (Gardner, 1993), formula un razonamiento apoyado en dos ejes que interactúan de la siguiente manera: por una parte, la ya mencionada teoría de las inteligencias múltiples y una aproximación conceptual a la creatividad que denomina perspectiva interactiva; por otra, en este segundo eje se reconocen tres niveles de análisis que no pueden ser desatendidos en una consideración de la creatividad: La persona con su propio perfil de capacidades y valores, el campo o disciplina en que trabaja con sus sistemas simbólicos característicos, y el ámbito circundante, con sus expertos, mentores, rivales y discípulos, que emiten juicios sobre la validez y calidad tanto del propio individuo como de sus productos. Conforme a esta perspectiva, la creatividad no puede ser interpretada situándose en forma exclusiva en alguno de estos niveles. Debe entenderse en todo momento como un proceso que resulta de una interacción, frecuentemente asincrónica, en la que participan los tres elementos. Al final, no se entiende por qué el libro se llama Mentes Creativas, dado que precisamente se intenta demostrar que la inteligencia y la creatividad no se alojan en la mente de forma exclusiva.

Dice el autor que no importa cuánto talento tenga una persona, y que no estaremos en condiciones de decidir su nivel de creatividad, a menos que no examinemos el modo o procedimiento mediante el cual se apropia de su campo, transformándolo o incluso creando uno nuevo; y no conocemos las relaciones con su ámbito, sus tensiones y conflictos.

Un punto interesante es el uso que hace el autor del concepto de inteligencia para referirse a una amplia variedad de capacidades humanas. Desde luego nadie deja de reconocer la existencia de la creatividad en la música o en la plástica, las sorprendentes habilidades del cuerpo, el liderazgo o el trabajo en equipo, pero agrupar todo esto bajo la misma categoría es una decisión polémica. Como se ha explicado, preferentemente el concepto de inteligencia se ha reservado para cuestiones asociadas al lenguaje y los números. Otras capacidades humanas han tenido el carácter de talento, habilidad, competencia, destreza, ingenio, pero en ningún caso de inteligencia.

Para Gardner el prerrequisito necesario de una teoría de la inteligencia, precisamente, es que abarque una gama razonablemente amplia y completa de las capacidades humanas presentes en distintas culturas. Es preciso dar cuenta de las habilidades de un chamán y un psicoanalista al igual que de un yoghi y un santo. No hay nada mágico en la palabra inteligencia. 
Se la ha escogido deliberadamente para entrar en la discusión de las capacidades humanas intentando romper el sesgo dominante.

Cada inteligencia expresa una capacidad que opera de acuerdo con sus propios procedimientos, sistemas y reglas, y tiene sus propias bases biológicas. Desde el punto de vista teórico, éste es el referente clave para resolver sobre el estatus de inteligencia de una capacidad. En síntesis, inteligencia es un vocablo útil para designar una experiencia o un fenómeno, en ningún caso es una entidad tangible y mensurable. Surge como una opción conceptual. En el contexto de la interminable polémica sobre la naturaleza humana y la educación, siempre habrá espacio para nuevas propuestas, y lo que se postula con unos fundamentos podrá cuestionarse con otros igualmente legítimos. Lo central está aquí en la opción de elevar a la categoría de inteligencia un conjunto variado de capacidades. No cabe insistir en que esta palabra nombra una característica positiva, y como pocas altamente valorada. Contribuir a producir una percepción en un marco de equivalencia para capacidades tan diversas no es trivial. Con ello se ha establecido un principio de igualdad que tiene como base el reconocimiento y aceptación de la diversidad.

La teoría de las inteligencias múltiples no tiene relación exclusivamente con razonamientos, evidencias científicas, acopio de datos y reflexiones, se encuentra en ella una concepción elevada de la diversidad humana.

Para acabar podemos decir que aunque la existencia de estas ocho inteligencias haya sido demostrada, no ha sido probado qué tipo de relación existiría entre ellas y la creatividad ¿será verdad que las ocho inteligencias son distintas?, ¿existen entonces ocho tipos de creatividad diferentes? o, más bien, ¿el perfil general obtenido con el alumno se relacionará con una medida general de la creatividad? Obviamente, si quisiéramos estudiar esto, tendríamos dos opciones: a) buscar una prueba de creatividad para cada inteligencia. Ya existen diversas pruebas de creatividad, generalmente en cuanto a creatividad verbal y creatividad gráfica. Pero faltarían por diseñar otras cinco pruebas; y b) otra opción sería correlacionar los resultados obtenidos por el alumno en las IM con una medida única de creatividad. ¿Qué podría obtenerse con esto? ¿Podría verse si la creatividad es general o está directamente relacionada con las inteligencias? Ciertamente la prueba de Torrance que utilizamos tiene un carácter grafico, con lo cual, sería de esperar que en nuestro estudio hubiera una mayor correlación entre 
creatividad y la inteligencia viso espacial. Esta es una de las hipótesis que planteamos en nuestro trabajo empírico, que pasamos a comentar.

El objetivo general del trabajo es estudiar la relación existente entre inteligencia y creatividad. Para ello, partiremos de dos enfoques o perspectivas diferentes de entender la inteligencia que van acompañadas de formas distintas de medirla. La perspectiva tradicional o psicométrica y una nueva perspectiva basada en la evaluación dinámica y multidimensional.

En términos generales en nuestro estudio se plantean dos fases:

Durante la primera se procede, por un lado, a medir la inteligencia mediante una prueba psicométrica estandarizada de carácter multifactorial pero que se asienta en el concepto de Cociente Intelectual (CI). Por otro lado, se lleva a cabo una valoración de la competencia cognitiva utilizando el modelo de evaluación diseñado por Gardner, Feldman y Krechervsky (1998 a, b y c) y que se fundamenta en un modelo multidimensional y funcional de entender y explicar la inteligencia, que formula la independencia entre las aptitudes intelectuales, acentuando el carácter constructivo de las mismas.

La segunda fase está destinada a la valoración de la creatividad utilizando para ello el test de pensamiento creativo de Torrance (TTCT) en su forma A y en la versión "muestre su imaginación con dibujos", que mide las variables de fluidez, flexibilidad, originalidad, y elaboración (Torrance, 1966, 1974).

A partir del contexto teórico analizado en los apartados anteriores, derivamos los siguientes objetivos específicos de nuestra investigación centrados en:

1. Establecer la relación entre el cociente intelectual general obtenido mediante la Batería de Aptitudes Diferenciales y Generales de Yuste (1998) y la creatividad valorada mediante el TTCT.

2. Determinar la relación existente entre las puntuaciones obtenidas por los alumnos en las actividades de evaluación de las IM y la creatividad.

3. Analizar los supuestos de la teoría del umbral planteada por Torrance. 


\section{Método}

\section{Participantes y centros}

La investigación ha sido realizada con 294 alumnos de $3^{\circ}$ de Educación Infantil y $1^{\text {o }}$ y $2^{\circ}$ de Educación Primaria pertenecientes a 3 centros educativos de las provincias de Murcia y Alicante. Dos de los centros son urbanos y uno semiurbano. Las características socioeconómicas de las familias de los alumnos de estos centros abarcan el rango típico.

\section{Instrumentos}

Los instrumentos empleados en esta investigación han sido en su mayor parte adaptados por los miembros de nuestro grupo de investigación, a partir de los utilizados por Gardner y colaboradores en el Proyecto Spectrum (1998, a, b y c).

\section{Batería de Aptitudes Diferenciales y Generales}

El objetivo es establecer los perfiles cognitivos de los alumnos respecto a la Inteligencia General, razonamiento analógico, memoria, atención y relaciones espaciales (Yuste, Martínez Arias y Galve, 1998).

Se han utilizado diferentes niveles de la batería BADyG, que se corresponden con las edades de los alumnos. Así, para los alumnos de Educación Infantil se utiliza BADyG I (edición renovada) y para los de $1^{\circ}$ y $2^{\circ}$ de Educación Primaria se emplea BADyG E1 (edición renovada). Estas pruebas permiten hallar el CI referido a la inteligencia general del alumno, así como puntuaciones parciales sobre factores verbales, numéricos y espaciales.

\section{Actividades de evaluación de las Inteligencias Múltiples}

Para la evaluación de las Inteligencias Múltiples (IM) se utilizan once actividades diseñadas por Gardner y colaboradores (1998c) en el Proyecto Spectrum. Las actividades han sido adaptadas y contextualizadas a nuestro contexto por miembros del grupo de investigación (Ferrándiz, 2003; Prieto y Ballester, 2003). Queremos señalar que las inteligencias Inter e intrapersonal no se han valorado en este estudio. 
El objetivo de las mismas es evaluar las habilidades implícitas en cada una de las inteligencias: lingüística; lógico-matemática; viso-espacial; corporal-cinestésica; naturalista y musical. Para cada una de las actividades los observadores cuentan con protocolos o escalas de observación tipo likert (puntuaciones que oscilan entre 1 a 4), en las que aparecen las habilidades de cada una de las inteligencias, objeto de evaluación. A continuación, presentamos de forma breve estos instrumentos de evaluación utilizados en este estudio empírico.

- Inteligencia naturalista: Para la evaluación de esta inteligencia se utilizan las actividades de "descubrimiento" y la de "flotar y hundir". Todas destinadas a evaluar las habilidades de observación precisa, identificación de relaciones, formulación y comprobación de hipótesis, experimentación, interés por actividades de la Inteligencia Naturalista y conocimiento del mundo natural.

- Inteligencia viso-espacial: La evaluación de la inteligencia viso-espacial se desarrolla en dos sesiones con las siguientes actividades estructuradas: crear una escultura, dibujar un animal, dibujar una persona y dibujar un animal imaginario. Se pretende evaluar las habilidades de representación, exploración, y talento artístico.

- Inteligencia corporal-cinestésica: Para la evaluación de esta inteligencia se utiliza la actividad llamada "movimiento creativo", destinada a valorar las habilidades de sensibilidad al ritmo, expresividad, control corporal, y generación de ideas mediante movimiento.

- Inteligencia lingüística: La inteligencia lingüística se desarrolla en dos sesiones, una para la actividad del "cuentacuentos" y otra para la del "reportero", ambas destinadas a valorar las habilidades siguientes: funciones primarias del lenguaje; habilidades de narración; y habilidades referidas a la información (nivel de andamiaje, precisión del contenido, estructura del argumento, complejidad de vocabulario, nivel de detalles y estructuración de las frases).

- Inteligencia lógico-matemática: Esta inteligencia es evaluada mediante la actividad del "juego del dinosaurio", destinada a valorar las habilidades de: razonamiento numérico, razonamiento lógico y razonamiento espacial.

- Inteligencia musical: La inteligencia musical es evaluada con la actividad del "canto" que pretende evaluar las habilidades de sensibilidad al tono, al ritmo y la capacidad musical. 


\section{Test de pensamiento creativo de Torrance (TTCT)}

El objetivo del test es evaluar la creatividad de niños. Hemos utilizado la forma figurativa. Los alumnos dan múltiples respuestas a estímulos, que son puntuados según la fluidez (o el número de ideas); la flexibilidad, con respecto a la variedad de las perspectivas representadas en las ideas; la originalidad (la infrecuencia estadística) y la elaboración de las ideas más allá de lo requerido por el estímulo. El TTCT en su forma figurativa consta de tres subtests o juegos: a) componer un dibujo, b) acabar un dibujo, c) componer diferentes realizaciones utilizando líneas paralelas.

En el primero, componer un dibujo, se pide al niño que realice un dibujo a partir de una forma dada, consistente en un trozo de papel adhesivo de color. Las habilidades que se valoran con este primer subtest son: a) originalidad, consistente en considerar las respuestas novedosas, no familiares e inusuales; y b) elaboración, se refiere a la cantidad de detalles que el niño añade al dibujo con el objetivo de embellecerlo.

El segundo subtest, acabar un dibujo, consta de 10 trazos, a partir de los cuales el niño tiene que utilizarlos realizando diferentes dibujos y poniéndoles un título. Se evalúa la elaboración (número de detalles añadidos al dibujo), la originalidad (respuestas inusuales y poco convencionales), la flexibilidad (variedad de categorías en las respuestas) y en menor grado la fluidez (número de dibujos con título realizados).

El tercer subtest, las líneas paralelas, consta de 30 pares de líneas paralelas. El objetivo es hacer tantos dibujos como se pueda utilizando los 30 pares. Se mide la fluidez (aptitud para hacer asociaciones múltiples a partir de un estímulo único); la flexibilidad, originalidad y elaboración (Torrance, 1974; Prieto, López y Ferrándiz, 2003).

\section{Procedimiento}

En un primer momento se llevó a cabo la administración de la prueba de inteligencia BADyG. Esta aplicación se realizó en el contexto aula y los datos los recogen diferentes miembros del equipo, entrenados previamente para tal fin. 
El segundo momento consiste en evaluar cada una de las inteligencias. El orden de la evaluación de las inteligencias ha sido el siguiente: naturalista, corporal-cinestésica, visoespacial, lingüística, lógico-matemática y musical. En todas las actividades están presentes los profesores de cada grupo de alumnos e intervienen en el proceso de evaluación. La duración de cada actividad es de aproximadamente 60 minutos. Esta es la fase más compleja de toda la investigación, por cuanto que exige una gran formación y manejo del sistema de evaluación de las Inteligencias Múltiples.

En un tercer momento se aplicó la prueba de creatividad TTCT (The Torrance of Creative Thinking Test). Se hizo individualmente para los niños de Infantil y en pequeños grupos para el resto de los niños. La aplicación de dicha prueba la realizan también los miembros del equipo.

Por último, se procedió a la corrección de las pruebas y a la valoración de las actividades, utilizadas como instrumentos de evaluación de las IM; se realizaron el procesamiento de datos y su análisis estadístico; finalmente, se extrajeron y valoraron los resultados obtenidos y se elaboraron un apartado de discusión y conclusiones abierto a cualquier tipo de aportación y consideración que se establezca.

\section{Análisis de datos}

Los objetivos propuestos así como el procedimiento seguido requieren principalmente el empleo de una metodología de análisis descriptivo, correlacional y en algunos casos del análisis inferencial. Todos los análisis estadísticos se llevan a cabo mediante el programa SPSS/PC versión 11.1.

\section{Resultados}

En primer lugar, en las tablas 1, 2 y 3 se recogen los análisis descriptivos (medias y desviaciones típicas) acerca de los resultados obtenidos por los participantes en los distintos instrumentos de evaluación utilizados. En la tabla 1 aparecen las medias obtenidas para las diferentes variables del test de inteligencia (BADyG). Los valores oscilan entre 35.74 para el razonamiento lógico y 42.77 para el razonamiento verbal. Se observa, por un lado, que las medias obtenidas por la muestra de participantes se encuentran por debajo de la media de la 
población. Por otro lado, es preciso apuntar que las desviaciones típicas obtenidas son muy elevadas lo que indica que existe una gran dispersión entre las puntuaciones.

Tabla 1.- Estadísticos descriptivos de la Batería de Aptitudes Diferenciales y Generales, para la medida de la inteligencia.

\begin{tabular}{l|c|c|c|c} 
& Mínimo & Máximo & Media & Desv. típ. \\
\hline Nivel Cognitivo General & 1 & 97 & 35,84 & 29,135 \\
\hline Razonamiento Verbal & 1 & 99 & 42,77 & 26,845 \\
\hline Razonamiento Numérico & 0 & 99 & 37,85 & 31,412 \\
\hline Aptitud Espacial & 1 & 99 & 32,00 & 29,124 \\
\hline Razonamiento Lógico & 1 & 98 & 35,74 & 30,162 \\
\hline Memoria & 1 & 99 & 36,62 & 31,278 \\
\hline N válido (según lista) & & & & \\
\hline
\end{tabular}

En la tabla 2 se muestran las medias de las puntuaciones obtenidas por los alumnos en las actividades de evaluación de las IM. En dicha tabla se observa que los valores son muy parecidos entre sí, a excepción de la inteligencia matemática que presenta una media más elevada (3.53); esto puede ser debido a que la actividad utilizada para valorar la inteligencia lógica-matemática presente menos dificultad, tal y como Ballester (2004) corrobora en su estudio.

Hay que indicar que las desviaciones típicas arrojan valores pequeños, todos por debajo de .6.; en general, las desviaciones típicas de las diferentes inteligencias son muy parecidas entre sí.

Tabla 2. Estadísticos descriptivos de las Inteligencias Múltiples.

\begin{tabular}{l|c|c|c|c} 
& \multicolumn{1}{|c|}{ Mínimo } & Máximo & \multicolumn{1}{c}{ Media } & Desv. típ. \\
\hline NATURALISTA & 1.00 & 4.00 & 2.4327 & .49469 \\
\hline LINGÜÍSTICA & 1.00 & 3.67 & 2.0551 & .56171 \\
\hline CORPORAL & 1.25 & 3.75 & 2.7283 & .48145 \\
\hline VISO_ESP & 1.00 & 4.00 & 2.5644 & .58803 \\
\hline MUSICAL & 1.00 & 4.00 & 2.5268 & .48867 \\
\hline MATEMÁTICA & 1.00 & 4.00 & 3.5318 & .55005 \\
\hline N válido (según lista) & & & &
\end{tabular}

En la tabla 3 se recogen los estadísticos descriptivos (medias y desviaciones típicas) de las diferentes variables en cada uno de los juegos o subtests del TTCT. 
Para proceder a la interpretación de los resultados obtenidos, hemos de precisar que las puntuaciones máximas que podrían alcanzar los alumnos en las diferentes dimensiones valoradas son:

a) Según los criterios de valoración para el juego 1, la puntuación máxima en la variable originalidad es de 5 puntos, mientras que para la elaboración no existe límite. Los datos recogidos en la tabla 3 ponen de manifiesto que las medias en ambas variables son bajas.

b) En el juego 2, la máxima puntuación alcanzable en fluidez es de 10 puntos; la media alcanzada por los participantes ha sido 8.45, lo que indica que una gran proporción de alumnos obtienen puntuaciones muy elevadas, incluso el máximo. En el caso de la flexibilidad, el máximo alcanzable es 10; en este sentido la media del grupo es moderadamente alta $(6,93)$ al igual que la desviación típica $(2,268)$. Sin embargo, las variables originalidad (cuyo máximo se situaría en 20) y elaboración (sin límite de puntuación) son las que obtienen medias y desviaciones más bajas.

c) El juego 3 es el más complejo por sus características, ya que presenta mayor número de items para valorar las diferentes dimensiones. Los datos de la tabla 3 muestran medias muy bajas y desviaciones muy elevadas en todas las dimensiones. Hemos de resaltar que ninguno de los participantes ha obtenido en este juego las puntuaciones máximas alcanzables en las distintas dimensiones, lo que puede ser debido a las características de la muestra o a las propias de la creatividad.

Tabla 3. Estadísticos descriptivos de las variables fluidez, flexibilidad, originalidad y elaboración para cada uno de los juegos del test de creatividad.

\begin{tabular}{|c|c|c|c|c|c|}
\hline & 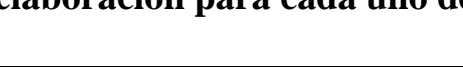 & Mínimo & Máximo & Media & Desv. típ. \\
\hline \multirow{2}{*}{ JUEGO 1} & ORIGINALIDAD & 0 & 5 & 1,27 & 1,685 \\
\hline & ELABORACIÓN & 0 & 11 & 3,07 & 2,165 \\
\hline \multirow{4}{*}{ JUEGO 2} & FLUIDEZ & 1 & 10 & 8,45 & 2,227 \\
\hline & FLEXIBILIDAD & 1 & 10 & 6,93 & 2,268 \\
\hline & ORIGINALIDAD & 1 & 20 & 9,06 & 3,554 \\
\hline & ELABORACIÓN & 0 & 29 & 5,15 & 4,840 \\
\hline \multirow{5}{*}{ JUEGO 3} & FLUIDEZ & 0 & 28 & 8,94 & 5,511 \\
\hline & FLEXIBILIDAD & 0 & 19 & 6,45 & 4,232 \\
\hline & ORIGINALIDAD & 0 & 54 & 11,87 & 9,342 \\
\hline & ELABORACIÓN & 0 & 27 & 3,15 & 5,311 \\
\hline & N VÁLIDO (SEGÚN LISTA) & & & & \\
\hline
\end{tabular}


Además de este primer acercamiento para ver el comportamiento de las diferentes variables del TTCT desde forma descriptiva, se procedió al análisis de la estructura interna del test. Los datos fueron sometidos a un análisis factorial exploratorio y los resultados indican lo siguiente: a) la existencia de cuatro factores que se corresponde de forma general con los juegos o subtests que componen la prueba, b) los factores quedan distribuidos de la siguiente manera: factor 1 (fluidez, flexibilidad y originalidad del juego 3); factor 2 (fluidez, flexibilidad y originalidad del juego 2); factor 3 (elaboración de los juegos 2 y 3 ) y factor 4 originalidad y elaboración del juego 1.

Con respecto a la fiabilidad, el coeficiente de correlación entre mitades (para el juego 3) alcanza un valor de .853 y un coeficiente de fiabilidad corregido mediante la fórmula de Spearman-Brown, entendido como la expresión de la consistencia entre dos mitades (teniendo en cuenta el juego en su conjunto), de .92. Lo cual indica una muy buena consistencia interna de este juego y aporta consistencia a los resultados obtenidos en el análisis factorial realizado.

\section{Creatividad e Inteligencia}

En la tabla 4 se presentan los análisis correlacionales llevados a cabo entre las variables de creatividad (fluidez, flexibilidad y originalidad) de los juegos $(1,2$ y 3$)$ y las variables que componen la Batería de Aptitudes Diferenciales y Generales.

Tabla 4. Coeficientes de correlación de Pearson entre las variables pertenecientes a cada uno de los factores que componen el TTCT

\begin{tabular}{|c|c|c|c|c|c|c|c|c|c|c|}
\hline & & Factor & & & Factor & & Facto & & Facto & \\
\hline & $\begin{array}{l}\text { Flu } \\
\text { (J.3) }\end{array}$ & $\begin{array}{l}\text { Fle } \\
(\text { J.3 })\end{array}$ & $\begin{array}{l}\text { Or } \\
(\mathbf{J} .3)\end{array}$ & $\begin{array}{l}\text { Flu } \\
(J .2)\end{array}$ & \begin{tabular}{|l|} 
Fle \\
(J.2)
\end{tabular} & $\begin{array}{l}\text { Or } \\
(J .2)\end{array}$ & \begin{tabular}{|l} 
Ela \\
(J. 2)
\end{tabular} & \begin{tabular}{|l|} 
Ela \\
(J. 3)
\end{tabular} & $\begin{array}{l}\text { Or } \\
\text { (J.1) }\end{array}$ & \begin{tabular}{|l|} 
Ela \\
$($ J.1 $)$
\end{tabular} \\
\hline $\begin{array}{l}\text { Nivel Cognitivo } \\
\text { General }\end{array}$ & $.126^{*}$ & .096 & .083 & .011 & -.006 & -.081 & .083 & $.129^{*}$ & .104 & .029 \\
\hline $\begin{array}{l}\text { Razonamiento } \\
\text { Verbal }\end{array}$ & $.122^{*}$ & .091 & .093 & .094 & .017 & .053 & -.006 & .032 & .102 & $.147^{*}$ \\
\hline $\begin{array}{l}\text { Razonamiento } \\
\text { Numérico }\end{array}$ & $.166^{* *}$ & $.147^{*}$ & .111 & .068 & .036 & \begin{tabular}{|l|}
-.009 \\
\end{tabular} & .020 & .013 & -.022 & \begin{tabular}{|l|}
-.098 \\
\end{tabular} \\
\hline $\begin{array}{l}\text { Aptitud } \\
\text { Espacial }\end{array}$ & .110 & .079 & .055 & .084 & .030 & .008 & .072 & $.134 *$ & $.165^{* *}$ & $.135^{*}$ \\
\hline $\begin{array}{l}\text { Razonamiento } \\
\text { Lógico }\end{array}$ & .098 & .088 & .097 & .046 & .000 & -.047 & .011 & .021 & .072 & -.003 \\
\hline Memoria & $.123^{*}$ & $.179 * *$ & $.120^{*}$ & .103 & $.235^{* *}$ & \begin{tabular}{|l|}
-.011 \\
\end{tabular} & .108 & -.075 & -.029 & .109 \\
\hline
\end{tabular}


Los coeficientes de correlación de Pearson entre las variables son muy bajos, lo que indica falta de relación entre la inteligencia valorada de forma psicométrica y las habilidades creativas. De hecho, si trabajamos con coeficientes de determinación (coeficientes de correlación al cuadrado), la información es mucho más fehaciente que la ofrecida por los anteriores, ya que indica la varianza compartida entre las variables correlacionadas. Así, para la correlación más elevada, la cual se da entre las variables de memoria y flexibilidad del juego 2, el coeficiente de determinación es de 0.05 , lo que indica que ambas variables comparten un 5\% de la varianza. Estos resultados están acordes con estudios que han examinado las correlaciones de las medidas de la creatividad con medidas psicometrías de inteligencia y que han encontrado relaciones moderadamente bajas (Holland, 1959; Torrance, 1962; Getzels y Jackson, 1962; Wallach y Kogan, 1965; MacKinnon, 1978; Davis y Rimm, 1977, 1979).

A continuación se presentan los coeficientes de correlación de Pearson entre los factores de creatividad extraídos y las diferentes inteligencias propuestas por Gardner (1983) en su modelo teórico. El objetivo es estudiar la existencia o no de relación entre las variables de creatividad que componen cada uno de los juegos o factores del TTCT y las inteligencias múltiples, valoradas mediante tareas abiertas, contextualizadas, dinámicas y significativas para los individuos, tal y como hemos explicado anteriormente.

Tabla 5. Coeficientes de correlación de Pearson entre las variables pertenecientes a cada uno de los factores que componen el TTCT y las Inteligencias Múltiples.

\begin{tabular}{l|l|l|l|l|l|l|l|l|l|l} 
& \multicolumn{3}{|c}{ FACTOR 1 } & \multicolumn{5}{c}{ FACTOR 2 } & \multicolumn{3}{|l}{ FACTOR 3 } & \multicolumn{2}{l}{ FACTOR } \\
\hline & fluidez & flex & ori & flui & flex & ori & ela & ela & orig & ela \\
& J. 3) & J. 3) & J. 3) & J. 2) & J. 2) & J. 2) & J. 2) & J. 3) & J. 1) & J.1) \\
\hline Naturalista & $250^{* *}$ & $180^{* *}$ & $163^{* *}$ & $151^{*}$ & 034 & 000 & $179^{* *}$ & .051 & 121 & 091 \\
\hline Lingüística & $228^{* *}$ & $204^{* *}$ & $166^{* *}$ & $160^{* *}$ & 063 & 056 & $182^{* *}$ & 026 & 107 & 111 \\
\hline Corporal & $196^{* *}$ & $156^{*}$ & 102 & $167^{* *}$ & 103 & 015 & $161^{* *}$ & .078 & 066 & 092 \\
\hline Viso- & $314^{* *}$ & $233^{* *}$ & $233^{* *}$ & $134^{*}$ & 021 & .033 & $157^{* *}$ & 044 & 111 & $252^{*}$ \\
\hline Mspacial & & & & & & & & & & $*$ \\
\hline Musical & 032 & 005 & .012 & .036 & .026 & .093 & 093 & 075 & 010 & 000 \\
\hline Matemática & $338^{* *}$ & $283^{* *}$ & $263^{* *}$ & $186^{* *}$ & 117 & 010 & $227^{* *}$ & 038 & $138^{*}$ & 114
\end{tabular}


Podemos decir que las correlaciones entre variables son bajas, aunque son algo mayores las relaciones entre las IM y la creatividad, que las obtenidas entre la creatividad y la inteligencia psicométrica.

Son las variables (originalidad, fluidez y flexibilidad) del factor 1 las que más relación tienen con las distintas inteligencias. Especialmente, se observa una mayor relación entre la inteligencia matemática y dichas variables, siendo la varianza que comparten $10 \%, 8 \%$ y $7 \%$ respectivamente.

En investigaciones anteriores hemos corroborado que el juego 3 (factor 1) es el más fiable de la prueba de creatividad (Ferrando, 2004; Ferrando, Ferrándiz, y Bermejo, 2005; Bermejo, Ferrándiz, y Prieto, 2005). Es este el juego que tiene una mayor relación con las inteligencias, siendo la capacidad para ofrecer múltiples soluciones a un problema la que más se relaciona con la perspectiva multidimensional de la inteligencia mantenida por Gardner (1983) y Gardner y col. (1998 a, b y c).

Dichos resultados nos llevan a establecer algunas posibles interpretaciones. En primer lugar, se corroboran las tesis que defienden la poca relación entre el constructo de creatividad y el de inteligencia.

En segundo lugar, parece ser también cierto que la relación entre dichos constructos depende de la conceptualización teórica de los mismos y su medida. Como se refleja en los resultados, las relaciones entre creatividad e inteligencias múltiples son más elevadas que las relaciones entre creatividad e inteligencia psicométrica.

Estos resultados reflejan la tesis defendida por Torrance (1962) y otros autores, quienes aluden a la escasa relación entre creatividad e inteligencia. Sin embargo, es preciso destacar que el autor también propone la teoría del umbral: un cierto nivel intelectual es condición necesaria (aunque no suficiente) para el desarrollo de la creatividad, esto es, la relación entre creatividad e inteligencia será mayor en determinados niveles intelectuales.

Con objeto de comprobar si la teoría del umbral se verifica en este estudio, procedemos a realizar un análisis de diferencia de medias en función del nivel intelectual. Para ello, 
se ha dividido la muestra en dos grupos: alumnos con alta inteligencia, aquellos que obtienen un CI igual o por encima de una desviación típica; alumnos con media-baja inteligencia, aquellos que obtienen un CI por debajo de una desviación típica. A partir de estos dos grupos, se ha estudiado si existen diferencias significativas entre ambos en las diferentes variables que componen el test de pensamiento creativo.

Los datos muestran que los alumnos con un mayor nivel intelectual obtienen puntuaciones más elevadas y estadísticamente significativas sólo en la variable elaboración del juego 3 ( $\mathrm{t}=2,247$, sig=,027). Estos resultados no apoyan la teoría del umbral establecida por Torrance (1962). Al parecer la relación entre creatividad e inteligencia se sitúa alrededor de un CI de 120, por lo que sería preciso un estudio más profundo de los alumnos con alto CI.

A continuación procedemos a analizar si la teoría del umbral se cumple para las IM. Los datos procedentes de los análisis realizados por Ferrando (2004) muestran que los alumnos con una alta inteligencia naturalista obtienen puntuaciones superiores en las diferentes variables de creatividad; dichas relaciones son significativas para las variables que forman el factor 1: fluidez ( $\mathrm{t}=-3.194$, sig $=, 002)$; flexibilidad $(\mathrm{t}=-1.897$, $\operatorname{sig}=, 059)$ y originalidad $(\mathrm{t}=-$ 2.433, sig=,016). También existen diferencias significativas en cuanto a alta inteligencia naturalista y la puntuación obtenida en la variable de elaboración $(\mathrm{t}=-2.119$, sig=.039) del juego 2.

Respecto a la inteligencia lingüística, podemos decir que el grupo de niños que obtienen medias superiores también obtienen puntuaciones superiores en todas las variables del test de creatividad, a excepción de la originalidad del juego 2. Estas diferencias son estadísticamente significativas en el factor 1 (fluidez $\mathrm{t}=-3.354$, sig=.001; flexibilidad $\mathrm{t}=-2.465$, sig=.014; y originalidad $\mathrm{t}=-1.970$, sig= .05); asimismo, en el factor 2 la significación se da para la variable fluidez ( $\mathrm{t}=-1.995$, sig=0.47); mientras que en el factor 3 , la variable de elaboración es significativa $(\mathrm{t}=-2.265$, sig=0.27). Finalmente, podemos decir que en el factor cuatro, la variable de elaboración arroja índices de significación $(\mathrm{t}=-2.122$, sig=0.35). 
En cuanto a la inteligencia corporal, las medias de los alumnos con alta inteligencia son superiores en todo el test de creatividad, a excepción de las variables de originalidad y elaboración de los juegos 2 y 3 respectivamente.

Sin embargo, las diferencias significativas se aprecian únicamente en el factor 1 (flui$\operatorname{dez} \mathrm{t}=-3.910$, sig=.000; flexibilidad t=-3.560, sig=.000; y elaboración $\mathrm{t}=-2.261$, sig=.025).

Las medias obtenidas por los alumnos con alta inteligencia viso-espacial son superiores en todas las variables de la prueba, a excepción de la elaboración del juego 3 en la que se aprecian medias muy similares entre ambos grupos, siendo tales diferencias estadísticamente significativas para el factor 1 (fluidez $\mathrm{t}=-4.260$, sig=.000; flexibilidad $\mathrm{t}=-3.282$, sig=.001; $\mathrm{y}$ originalidad $\mathrm{t}=-3.827$, $\mathrm{sig}=.000)$, y para la variable de elaboración del factor $1(\mathrm{t}=-3.409$, $\operatorname{sig}=.001)$.

Por último, observamos que son los alumnos con una inteligencia musical por debajo de $1 \mathrm{z}$ los que obtienen en general medias superiores, a excepción de la variable flexibilidad del factor 2, siendo las diferencias significativas para la variable fluidez del juego 1 (factor 3 ) $(\mathrm{t}=1.920, \operatorname{sig}=.056)$.

Es preciso apuntar que el análisis de la inteligencia lógico-matemática no se ha podido realizar debido a que todos los alumnos obtenían puntuaciones en dicha inteligencia iguales o por encima de una desviación típica.

En síntesis, podemos decir que los alumnos que puntúan alto en la mayoría de las inteligencias múltiples (naturalista, lingüística, corporal y viso-espacial) son también aquellos que obtienen puntuaciones significativamente superiores a los del grupo de habilidades medias / bajas en las variables de originalidad, fluidez y flexibilidad del factor 1.

Ante este resultado, hemos realizado un gráfico donde presentamos las medias obtenidas por cada uno de los grupos que han puntuado alto en cada una de las inteligencias, y las puntuaciones referidas a la fluidez, flexibilidad y originalidad del factor uno de creatividad. 


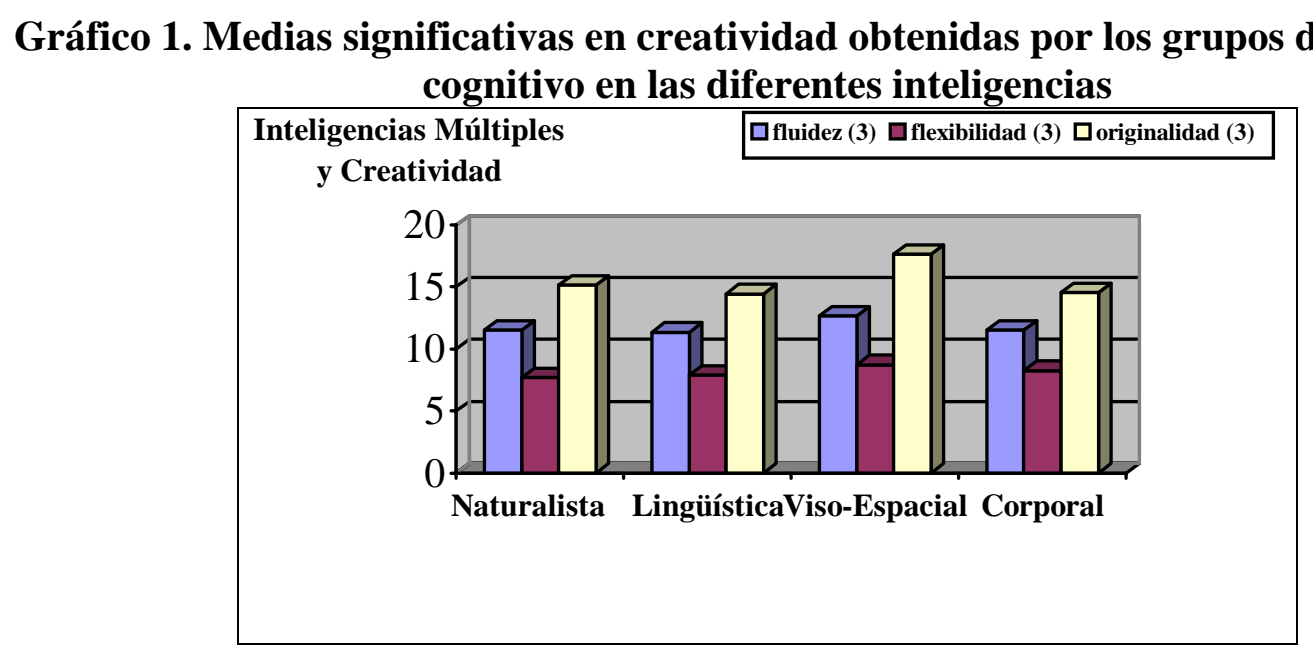

En el gráfico se observa que los alumnos con una elevada inteligencia viso-espacial obtienen puntuaciones superiores en fluidez, flexibilidad y originalidad, seguidos de los alumnos con alta inteligencia naturalista, corporal y lingüística respectivamente. Además, es preciso destacar que la originalidad es la variable que más difiere entre los distintos grupos de inteligencias, sería, por tanto, la variable que distingue más a los grupos con diferentes niveles intelectuales.

\section{Discusión y conclusiones}

En primer lugar, queremos resaltar que con el presente trabajo hemos profundizado en el estudio de la creatividad y de su relación con la inteligencia. Dicha relación se ha establecido utilizando dos perspectivas diferentes de entender la inteligencia: una, la psicométrica tradicional, que nos ofrece una medida general de la inteligencia; la otra, la multidimensional de Gardner, que nos ofrece medidas independientes de inteligencia naturalista, lógica, linguiística, musical, viso-espacial y corporal.

Segundo, los resultados indican bajas relaciones entre creatividad e inteligencia. No obstante, la relación entre ellos cambia según se conceptualice el constructo de inteligencia. En general, podríamos decir que existe una mayor relación entre la creatividad y las inteligencias múltiples, de hecho este resultado es obvio pues las tareas de evaluación de las IM son mucho más abiertas y dinámicas que las del BADyG, en términos de Guilford la medida de evaluación del BADyG se centra en el pensamiento convergente (una única solución correc- 
ta), mientras que las de Gardner permiten en mayor medida la utilización del pensamiento divergente (varias soluciones pueden ser correctas para un mismo problema)

Tercero, no se constata la teoría del umbral cuando se relaciona el CI con la creatividad: los alumnos con un mayor Cociente Intelectual no son los más creativos. Sin embargo, desde la perspectiva multidimensional la teoría del umbral se corrobora para la inteligencia viso-espacial, corporal, naturalista y lingüística; es decir, los alumnos que obtienen puntuaciones más elevadas en la competencia cognitiva referente a un determinado ámbito, obtienen a su vez puntuaciones más elevadas en el factor 1 de creatividad. Es el grupo que alcanza una alta inteligencia viso-espacial el que en mayor medida corrobora la teoría del umbral, pues son los más creativos; aunque hemos de precisar que el Torrance utilizado ha sido el figurativo.

Cuarto, nos gustaría añadir que tras todos los estudios llevados a cabo, el factor 1 representado por las variables de fluidez, flexibilidad, y originalidad y valorado mediante una tarea que requiere ofrecer múltiples, variadas y novedosas soluciones ante 30 pares de líneas paralelas (un mismo estímulo una y otra vez), se constituye como el más consistente y que aporta un mayor conocimiento sobre la relación existente entre creatividad e inteligencia.

Finalmente, quisiéramos señalar la necesidad de diseñar instrumentos específicos para valorar la creatividad dentro de cada dominio o inteligencia.

\section{Referencias}

Alonso, M. (2000). ¿Qué es la creatividad? Madrid: Biblioteca Nueva.

Ballester, P. (2004). Evaluar y atender la diversidad de los alumnos desde las inteligencias múltiples. Tesis doctoral. Universidad de Murcia.

Barron, F. (1988). Putting creativity to work. En R. J. Sternberg (ed.). The nature of creativity: Contemporary psychological perspectives (pp. 76-98). Cambridge: Cambridge University Press

Barron, F. y Harrington, D. (1981). Creativity, intelligence and personality. En M.R. Rosenzweig y L.W. Porter (eds.) Annual Review of Psychology, Vol. 32, 439-476. CA. Palo Alto: Annual Review. 
Bermejo, M.R; Ferrándiz, C. y Prieto, M.D. (2005). An empirical aproximation to the creative consistency during the creative. The First International Conference "Creativity: A Multifaceted View”. Moscow: 15-18, Septiembre 2005

Davis, G.A. y Rimm, S. (1977). Characteristics of creatively gifted children. Gifted Child Quaterly, December, 21.

Davis, G.A. y Rimm, S. (1979). GIFFI I and II. Group inventories for finding interests. Watertown, Wl: Educational Assessment Service.

Ferrándiz, C. (2003). Evaluación y desarrollo de la competencia cognitiva. Un estudio desde el modelo de las inteligencias múltiples. Tesis Doctoral. Universidad de Murcia (Premio Nacional de Investigación Educativa 2004, pendiente de publicación MEC).

Ferrando, M. (2004). Creatividad e inteligencias múltiples. Tesina de Licenciatura. Universidad de Murcia.

Ferrando, M., Ferrandiz, C. y Bermejo, M.R. (2005). Paper: Intelligence and creativity. The First International Conference "Creativity: A Multifaceted View" Moscow: 15-18, Septiembre 2005.

Gardner, H. (1983). Frames of mind. New York: Basic Books. (Traducción castellana, Estructuras de la mente. La teoría de las Inteligencias Múltiples. México: Fondo de Cultura Económica, 1987. Última Edición 2001).

Gardner, H. (1993). Creating minds: An anatomy of creativity. New York: Basic Books. (Traducción castellana, Mentes creativas. Barcelona: Paidós, 1997).

Gardner, H., Feldman, D. y Krechevsky, M. (1998a). Project Spectrum: Building on Children's Strengths: The Experiene of Project Spectrum. N. Y.: Teachers College Press. (Traducción castellana, El Proyecto Spectrum. Tomo I: Construir sobre las capacidades infantiles. Madrid: Morata, 2000).

Gardner, H., Feldman, D. y Krechevsky, M. (1998b). Project Spectrum: Early Learning Activities. N. Y.: Teachers College Press. (Traducción castellana, El Proyecto Spectrum. Tomo II: Actividades de aprendizaje en Educación Infantil. Madrid, Morata, 2000).

Gardner, H., Feldman, D. y Krechevsky, M. (1998c). Project Spectrum: Preschool Assessment Handbook. N. Y.: Teachers College Press. (Traducción castellana, El Proyecto Spectrum. Tomo III: Manual de evaluación para la Educación Infantil. Madrid: Morata, 2000).

Genovard, C, Prieto, M.D., Bermejo, R., y Ferrándiz, C. (en prensa) The History of creativity in Spain. NY: Cambridge University Press. 
Getzels, J. W. y Jackson, P. W. (1962). Creativity and intelligence: Explorations with gifted students. New York: Wiley.

Guilford, J.P. (1950). Creativity. American Psychologist 5, 444-454.

Holland, J.L. (1959). Some limitations of teacher ratings as predictors of creativity. Journal of Educational Psychology, 50, 219-223

Mackinnon, D.W. (1962). What makes a person creative? Saturday Review, 46-69.

Mackinnon, D.W. (1970). The personality correlates of creativity: A study of American architecs. En P.E. Vernon (Ed.), Creativity (pp.289-311). Harmondsworth: Penguin

MacKinnon, D. W. (1978). In search of human effectiveness: Identifying and developing creativity. Buffalo, NY: Creative Education Foundation.

Mednick, S.A. (1963). The associative basics of the creative process. Psychological Review, 69, 202-232.

Prieto, M.D. y Ferrándiz, C. (2001). Inteligencias Múltiples y currículum escolar. Málaga: Aljibe.

Prieto, M.D. y Ballester, P. (2003). Las Inteligencias Múltiples. Diferentes formas de enseñar y aprender. Madrid: Pirámide.

Prieto, M.D., López, O. y Ferrándiz, C. (2003). La creatividad en el contexto escolar. Estrategias para favorecerla. Madrid: Pirámide.

Renzulli, J. (1976). New Directions in Creativity. Nueva York: Harper and Row

Renzulli, J. (1977). The enrichment triad model. Mandsfiel Center, CT: Creative Learning Press.

SPSS/PC 11.0 for Windows (2001). Statistical Package for the Social Sciences. SPSS Inc.

Sternberg, R.J. (1988). A three-facet model of creativity. En R. Sternberg (ed.) The nature of creativity. Contemporary psychological perspectives (pp.125-147). Cambridge, MA: Cambridge University

Sternberg, R. y O’Hara, L. (1999). Creativity and intelligence. En R. Sternberg (Ed.) Handbook of creativity. Nueva York: Cambridge University Press

Torrance, E.P. (1962). Guiding creative talent. Englewood Cliffs, NJ: Prentice Hall.

Torrance, E. P. (1966). The Torrance Tests of Creative Thinking - Norm Technical Manual Research Edition - Verbal Tests, Forms A and B - Figural Tests, Forms A and B. Princeton NJ: Personnel Press.

Torrance, E. P. (1974). The Torrance tests of creative thinking. Bensenville, IL: Scholastic Testing Service. 
Wallach, M.A. y Kogan, N. (1965). Modes of thinking in children. Nueva York: Holt, Rinehart y Winston.

Weisberg, R.W. y Alba, J.W. (1981). An examination of the alleged role of "fixation" in the solution of several "insight” problems. Journal of Experimental Psychology, 110, 169192.

Weisberg, R.W. (1988). Problem solving and creativity. En R. Sternberg (ed.) The nature of creativity. Contemporary psychological perspectives (pp.148-176). Cambridge, MA: Cambridge University

Yuste, C., Martínez, R. y Galve, J.L. (1998) BADyG. Manual Técnico. Madrid: CEPE. 
Inteligencia y creatividad

[Página en blanco por razones de paginación] 